\title{
Vortex Lines in Motion
}

\section{BIAŁYNICKI-BIRULA*}

Center for Theoretical Physics and Institute of Theoretical Physics

Warsaw University, Warsaw, Poland

\section{T. MŁODUCHOWskI}

S.I. Witkiewicz High School, Elbląska 51, 01-737 Warsaw, Poland

\section{T. RADOŻYCKI}

Department of Physics, Warsaw University, Hoża 69, 00-681 Warsaw, Poland

$$
\text { AND C. ŚLIWA }
$$

Center for Theoretical Physics, al. Lotników 32/46, 02-668 Warsaw, Poland

(Received November 15, 2001)

\begin{abstract}
We extend our previous analysis of the motion of vortex lines in wave mechanics to the case of more elaborate vortex patterns and to a rotating harmonic trap.
\end{abstract}

PACS numbers: 03.65.- $-\mathrm{w}, 67.40 . \mathrm{Vs}$

\section{Introduction}

The hydrodynamic formulation of wave mechanics discovered by Madelung [1] offers an opportunity to directly connect the motion of quantum particles with the motion of a fluid - the probability fluid. This formulation not only offers a different way to visualize the quantum mechanical evolution but also introduces new objects: quantized vortices. Vorticity field is a very interesting physical object already in the study of classical fluids but in the framework of wave mechanics it acquires a new feature. In the three-dimensional configuration space vorticity can generically exist only on vortex lines and, in addition, the circulation around each vortex line must be quantized in units of $2 \pi \hbar / \mathrm{m}$. The fascination with vortex

*corresponding author; e-mail: birula@cft.edu.pl 
lines goes back many centuries when Empedocles, Aristotle [2], and Descartes [3] tried to explain the formation of the Earth, its gravity, and the dynamics of the whole solar system as due to primordial cosmic vortices. Later Lord Kelvin [4] has attempted to describe atoms as vortex rings. The proper mathematical description of hydrodynamic vortices has been started by Helmholtz [5]. The best summary of the present day theory of vorticity in classical hydrodynamics may be found in a recent monograph by Saffman [6].

There are two reasons why we are interested in the study of vortices embedded in solutions of wave equations. First, vortices are by themselves very interesting structures that deepen our understanding of wave phenomena. Second, recent advances in experiments on the Bose-Einstein condensation made it possible to create vortices in the laboratory providing a testing ground for the theoretical analysis of the vortex motion. Surprisingly, despite the fact that one can find with relative ease the behavior of even fairly complicated vortex structures, there has been very little activity in that area of research. The notable example is the research carried out for a long time by Berry, Nye, and their collaborators [7-9]. We would like, however, to emphasize the differences between their approach and our approach. They stress the generic features of vortex lines and apply their analysis mainly to monochromatic waves in optics without any reference to time evolution. We restrict ourselves to quantum mechanics and we study in detail specific examples exhibiting the time evolution of vortices. Thus, we believe that these two complementary approaches supplement each other.

In our first publication [10] we have introduced a general method of generating solutions of the Schrödinger equation with embedded vortices of almost arbitrary complexity. In the second paper [11] we have extended the analysis to the nonlinear wave equation with harmonic interparticle forces. In the present paper we restrict ourselves to the linear Schrödinger equation but in order to see a more intricate behavior, not seen in the examples studied before, we extend the scope of our analysis to cover more elaborate vortex structures. We also study the case of a rotating harmonic trap that shows some new features and might also be of experimental interest.

\section{Generating function}

In contrast to the situation in classical hydrodynamics, vorticity in quantum mechanics cannot be present in a three-dimensional volume. Due to the requirement that the wave function be single valued, vorticity in quantum mechanics is generically concentrated on lines. Vortex lines are defined as an intersection of the two surfaces defined by the vanishing of the real and imaginary part of the wave function.

The construction of solutions of wave equations, studied in [10], that exhibit various vortex structures, was based on the idea of a generating function. As a 
generating function we may use any solution $\psi_{k}(r, t)$ of the Schrödinger wave equation under study that satisfies the initial condition

$$
\psi_{\boldsymbol{k}}(\boldsymbol{r}, t=0)=\exp (\mathrm{i} \boldsymbol{r} \cdot \boldsymbol{k}) \phi_{0}(\boldsymbol{r})
$$

where $k$ is an auxiliary wave vector and $\phi(r)$ is a smooth, non-vanishing function. By taking a linear combination of derivatives with respect to the components of the vector $\boldsymbol{k}$ of the generating function (1), we can produce an arbitrary polynomial in the variables $x, y$, and $z$ in front of this function,

$$
\left[W_{\mathrm{R}}(\boldsymbol{r})+\mathrm{i} W_{\mathrm{I}}(\boldsymbol{r})\right] \phi_{0}(\boldsymbol{r}),
$$

where $W_{\mathbf{R}}$ and $W_{\mathrm{I}}$ are two real polynomials. Vortex lines are defined by the equations

$$
W_{\mathrm{R}}(r)=0, \quad W_{\mathrm{I}}(r)=0 .
$$

In this manner we obtain an initial wave function that may contain all types of intricate vortex structures. The motion of the vortices is determined by the Schrödinger equation. Since the Schrödinger equation is linear and it does not involve $\boldsymbol{k}$, all derivatives of $\psi_{\boldsymbol{k}}(\boldsymbol{r}, t)$ with respect to the components of the vector $\boldsymbol{k}$ also satisfy this equation. These derivatives at $\boldsymbol{k}=\mathbf{0}$ in all known cases, when the solution of the Schrödinger equation can be written down explicitly, have the form

$$
\left[W_{\mathrm{R}}(r, t)+\mathrm{i} W_{\mathrm{I}}(r, t)\right] \phi_{0}(r, t),
$$

where now $W_{\mathrm{R}}$ and $W_{\mathrm{I}}$ have coefficients that in general depend on time. The time evolution of vortices is determined by the time evolution of the zeros of the wave function. This requires solving two simultaneous real algebraic equations

$$
W_{\mathbf{R}}(r, t)=0, \quad W_{\mathrm{I}}(r, t)=0 .
$$

It is worth stressing that the motion of vortices depends on the shape of the "envelope" wave function $\phi_{0}(\boldsymbol{r})$. The same vortices "sitting" on different envelope functions will move in a different way.

\section{Simple configurations of vortex lines and vortex rings}

We have used in [10] an example of two vortex lines moving according to the free-particle Schrödinger equation to exhibit the phenomenon of vortex reconnection. The dynamics of three vortex lines in free motion has already been described in our preliminary report [12] where we have shown that it exhibits novel features. Namely, the three vortex lines go through the reconnection process in a different way; the reconnection occurs through the creation of a closed ring that shrinks and disappears. Since the full solution of the Schrödinger equation is time reversal symmetric, for negative times the whole process occurs in a reversed order: the vortex ring suddenly appears, then grows, and finally it is swallowed by the three 
vortex lines. A similar creation of an additional vortex ring accompanies the vortex reconnection also for other configurations studied in this section: a vortex circle and a vortex line. We shall make the simplest choice of the generating function for the free-particle Schrödinger equation, viz., a plane wave

$$
\psi_{\boldsymbol{k}}(\boldsymbol{r}, t)=\exp (\mathrm{i} \boldsymbol{r} \cdot \boldsymbol{k}) \exp (-\mathrm{i} \boldsymbol{k} \cdot \boldsymbol{k t} / 2) .
$$

We use the units $\hbar=1, m=1$ throughout this paper with the only exception of final formulas (27)-(34) for the generating function in the rotating trap. The initial wave function describing three orthogonal vortex lines has the form (2), with the following choice of the initial polynomial

$$
W_{\mathrm{R}}(r, t=0)+\mathrm{i} W_{\mathrm{I}}(r, t=0)=(x-d+\mathrm{i} y)(y-d+\mathrm{i} z)(z-d+\mathrm{i} x) .
$$

The time-dependent polynomials obtained by differentiating the function (6) and setting $\boldsymbol{k}=\mathbf{0}$ are

$$
\begin{aligned}
& W_{\mathbf{R}}(r, t)=-d^{3}+t(3 d-x-y-z) \\
& \quad+d^{2}(x+y+z)-x y^{2}-y z^{2}-z x^{2}+x y z \\
& W_{\mathbf{I}}(r, t)=-t(x+y+z)+d^{2}(x+y+z) \\
& \quad-d\left(x^{2}+y^{2}+z^{2}+x y+y z+z x\right)+x z^{2}+y x^{2}+z y^{2}-x y z
\end{aligned}
$$

and the motion is depicted in Fig. 1.
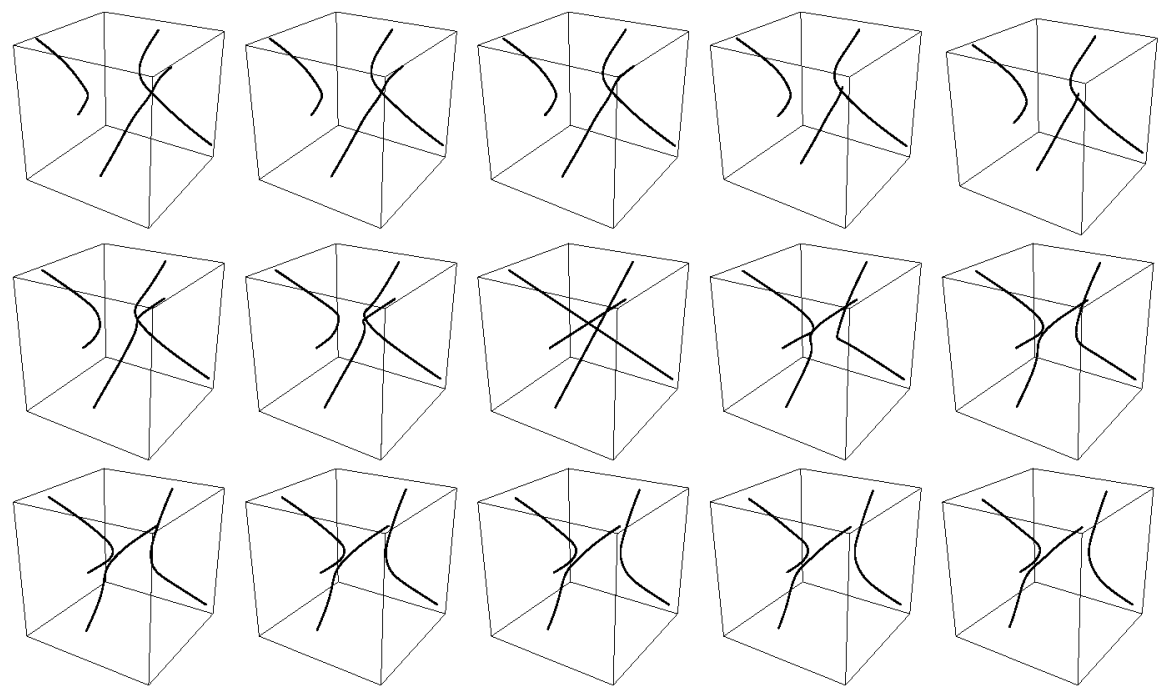

Fig. 1. Full history of the time evolution of three vortex lines that at $t=0$ are mutually perpendicular and nonintersecting. 
In the case of a line and a ring, the initial polynomial will be chosen as

$$
W_{\mathrm{R}}(\boldsymbol{r}, t=0)+\mathrm{i} W_{\mathrm{I}}(\boldsymbol{r}, t=0)=\left(x^{2}+y^{2}-R^{2}+\mathrm{i} R z\right)(x-d \pm \mathrm{i} z) .
$$

Depending on the sign in the second term the vorticities of the ring and the straight line are the same or opposite. The time dependent polynomials in this case are

$$
\begin{aligned}
& W_{\mathrm{R}}(r, t)=\mp 2 t z+(x-d)\left(x^{2}+y^{2}-R^{2}\right) \mp R z^{2} \\
& W_{\mathrm{I}}(r, t)=t(4 x \mp R-2 d) \pm\left(x^{2}+y^{2}-R^{2}\right) z-d R z+R x z .
\end{aligned}
$$

Finally, for the two rings we have chosen the initial polynomial in the form

$$
\begin{aligned}
& W_{\mathrm{R}}(r, t=0)+\mathrm{i} W_{\mathrm{I}}(\boldsymbol{r}, t=0)=\left(x^{2}+y^{2}-R^{2}-\mathrm{i} R z\right) \\
& \times\left[\left(x-x_{0}\right)^{2}+y^{2}-R^{2}+\mathrm{i} R\left(z-z_{0}\right)\right],
\end{aligned}
$$

that leads to the following time-dependent real and imaginary parts:

$$
\begin{gathered}
W_{\mathrm{R}}(r, t)=2 R z_{0} t-8 t^{2}+\left(x^{2}+y^{2}-R^{2}\right) \\
\times\left[\left(x-x_{0}\right)^{2}+y^{2}-R^{2}\right]+z\left(z-z_{0}\right), \\
W_{\mathrm{I}}(r, t)=t\left[8\left(x^{2}+y^{2}-x_{0} x\right)-3 R^{2}-2 x_{0}^{2}\right] \\
\quad+R z_{0}\left(x^{2}+y^{2}-R^{2}\right)-R z\left(x_{0}^{2}-2 x_{0} x\right) .
\end{gathered}
$$

In Figs. 2, 3, and 4 we show the most characteristic movie frames for three vortex lines, for a vortex line and a vortex ring (for upper signs in Eqs. (10a)), and for two vortex rings. In order to get a better view, we have tilted the axes.
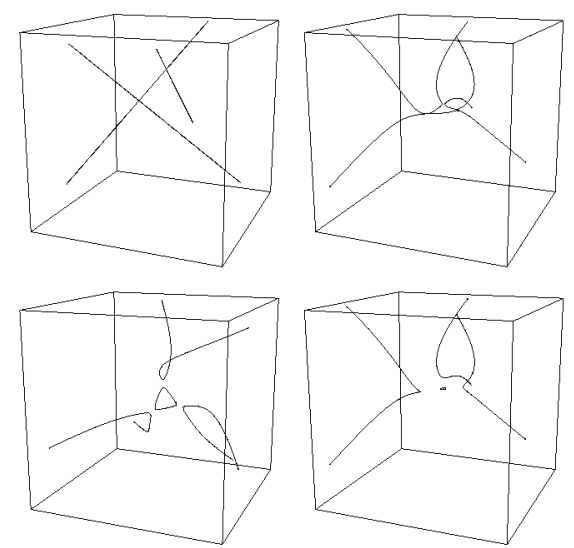

Fig. 2. The creation of a vortex ring by three vortex lines. 

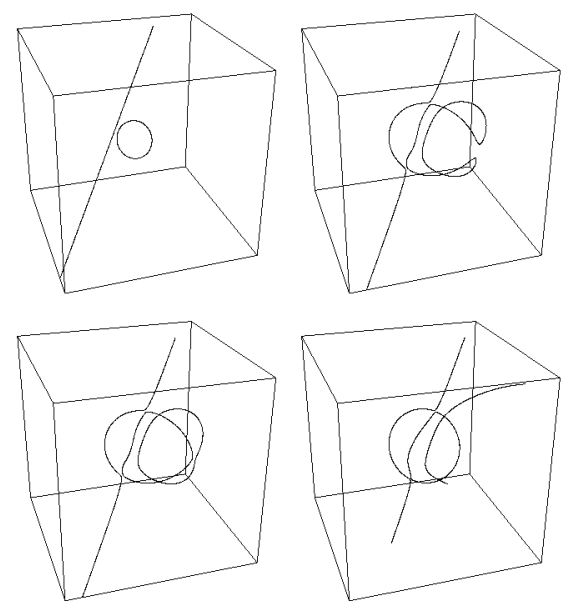

Fig. 3. A vortex ring and a vortex line create a second ring.
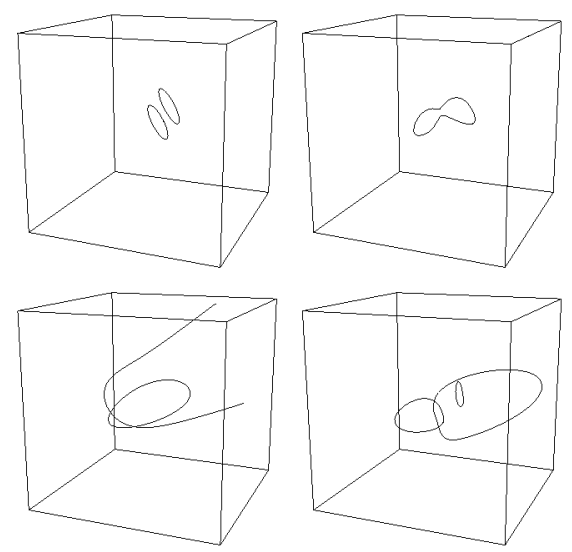

Fig. 4. Two vortex rings create a third ring.

\section{Rotating harmonic trap}

A general stationary harmonic trap centered at the origin of the coordinate system is described by a quadratic function of the coordinates $V(r)=(1 / 2) v_{i j} x^{i} x^{j}$. A rotating trap in the laboratory frame is described by the potential

$$
V(\boldsymbol{r}, t)=(1 / 2) v_{i j}(t) x^{i} x^{j}=V(\boldsymbol{r}(t)),
$$

where the time dependence of the matrix $v_{i j}(t)$ results from the rotation. In what follows we shall treat only a special case when the axis of rotation coincides with one of the principal directions of the trap. In this case, the time dependence of the potential has the form (we choose the $z$ axis as the axis of rotation) 


$$
\left[v_{i j}(t)\right]=\left[\begin{array}{cc}
\omega_{x}^{2} \cos ^{2}(\Omega t)+\omega_{y}^{2} \sin ^{2}(\Omega t) & \left(\omega_{x}^{2}-\omega_{y}^{2}\right) \cos (\Omega t) \sin (\Omega t) \\
\left(\omega_{x}^{2}-\omega_{y}^{2}\right) \cos (\Omega t) \sin (\Omega t) & \omega_{x}^{2} \sin ^{2}(\Omega t)+\omega_{y}^{2} \cos ^{2}(\Omega t)
\end{array}\right]
$$

Contour lines of this potential are the ellipses rotating in the positive (anticlockwise) direction in the $x y$ plane. The time dependence of the parameters of the potential may be replaced by the time dependence of the coordinates

$$
V(r, t)=V(r(t))
$$

In our case the time dependence of $r(t)$ is given by the formulae

$$
\begin{aligned}
& x(t)=x \cos (\Omega t)+y \sin (\Omega t), \\
& x(t)=-x \sin (\Omega t)+y \cos (\Omega t), \\
& z(t)=z .
\end{aligned}
$$

The functions $r(t)$ are the coordinates in the rotating frame where the trap is stationary. The Schrödinger equation for a rotating harmonic trap in the laboratory frame has the form

$$
\mathrm{i} \partial_{t} \psi(r, t)=\left[-\frac{1}{2} \Delta+V(r(t))\right] \psi(r, t)
$$

The time dependence of the Hamiltonian in this equation may be eliminated by the transformation to the rotating frame. The wave function $\psi(r, t)$ in the laboratory frame is related to the wave function $\phi(r, t)$ in the rotating frame through the formula

$$
\psi(r, t)=\phi(r(t), t)=\exp (-\mathrm{i} \boldsymbol{\Omega} \cdot \hat{\boldsymbol{M}} t) \phi(r, t),
$$

where $\hat{\boldsymbol{M}}$ is the angular momentum operator. This transformation cancels the time dependence of the potential since

$$
\begin{aligned}
& \exp (\mathrm{i} \boldsymbol{\Omega} \cdot \hat{\boldsymbol{M}} t) x(t) \exp (-\mathrm{i} \boldsymbol{\Omega} \cdot \hat{\boldsymbol{M}} t)=x \\
& \exp (\mathrm{i} \boldsymbol{\Omega} \cdot \hat{\boldsymbol{M}} t) y(t) \exp (-\mathrm{i} \boldsymbol{\Omega} \cdot \hat{\boldsymbol{M}} t)=y
\end{aligned}
$$

but it introduces an extra term into the Hamiltonian. Upon substituting the relation (18) into Eq. (17), we obtain

$$
\mathrm{i} \partial_{t} \phi(r, t)=\left[-\frac{1}{2} \Delta+V(r)-\boldsymbol{\Omega} \cdot \hat{\boldsymbol{M}}\right] \phi(r, t) .
$$

The additional term $-\boldsymbol{\Omega} \cdot \hat{\boldsymbol{M}}$ is responsible for the centrifugal force and the Coriolis force that act in the rotating frame. The generating function $\phi_{k}(r, t)$ for all polynomial vortex structures built on the fundamental, Gaussian state wave function in a trap in the rotating frame is the solution of the Schrödinger equation (17) satisfying the initial condition

$$
\phi_{\boldsymbol{k}}(\boldsymbol{r}, t=0)=\exp (\mathrm{i} k \cdot r) \phi_{0}(r) .
$$


In the general case, for an arbitrary rotation, the wave function $\phi_{0}(r)$ and hence also $\phi_{\boldsymbol{k}}(\boldsymbol{r}, t)$ cannot be explicitly written down since the set of algebraic equations for the coefficients of the Gaussian wave function cannot be solved in a closed form. However, in a special case considered in this paper, when the angular velocity is aligned with one of the symmetry axes of the trap, the general solution is relatively simple. In this case the motion in the $z$ direction uncouples from the motion in the $x y$ plane and the generating function factorizes into a product. The part of the generating function describing the motion in the $z$ direction does not involve the rotation and it has been already written down in Ref. [10]. Thus, the three-dimensional problem reduces to the solution of the Schrödinger equation in a rotating anisotropic harmonic trap in two dimensions. In what follows we shall choose the coordinate axes $x$ and $y$ along the main axes of the potential and for definitness we assume that $\omega_{x}>\omega_{y}$.

The solution of the equations of motion for the harmonic oscillator in a rotating frame in classical and in quantum theory involves two characteristic frequencies $\omega_{+}$and $\omega_{-}$given by the formulas (cf., for example, [13])

$$
\begin{gathered}
\omega_{+}=\frac{\sqrt{2 \Omega^{2}+\omega_{x}^{2}+\omega_{y}^{2}+\sqrt{\left(\omega_{x}^{2}-\omega_{y}^{2}\right)^{2}+8 \Omega^{2}\left(\omega_{x}^{2}+\omega_{y}^{2}\right)}}}{\sqrt{2}}, \\
\omega_{-}=\frac{\sqrt{2 \Omega^{2}+\omega_{x}^{2}+\omega_{y}^{2}-\sqrt{\left(\omega_{x}^{2}-\omega_{y}^{2}\right)^{2}+8 \Omega^{2}\left(\omega_{x}^{2}+\omega_{y}^{2}\right)}}}{\sqrt{2}} .
\end{gathered}
$$

The requirement that the expression under the outer square root for $\omega_{-}$be positive leads to the following condition:

$$
\left(\Omega^{2}-\omega_{x}^{2}\right)\left(\Omega^{2}-\omega_{y}^{2}\right)>0 .
$$

We infer from this that there are two regions of stable oscillations separated by a gap: slow rotations $\Omega<\omega_{y}<\omega_{x}$ and fast rotations $\omega_{y}<\omega_{x}<\Omega$. In the slow rotation regime the trapping forces are stronger than the centrifugal force. In the fast rotation regime the centrifugal force overwhelms the trapping forces but nevertheless stability holds due to the action of the Coriolis force. This is the same stabilization mechanism as in the Paul trap or in the Trojan states of electrons (cf. $[13,14]$ ). It turns out that in the investigation of the solutions of the Schrödinger equation it is convenient to work not with $\omega_{+}$and $\omega_{-}$but with their linear combinations $\Omega_{1}$ and $\Omega_{2}$

$$
\Omega_{1}=\frac{\sqrt{2 \Omega^{2}+\omega_{x}^{2}+\omega_{y}^{2}+2 \epsilon \sqrt{\left(\omega_{x}^{2}-\Omega^{2}\right)\left(\omega_{y}^{2}-\Omega^{2}\right)}}}{2}=\frac{\omega_{+}+\epsilon \omega_{-}}{2}
$$




$$
\Omega_{2}=\frac{\sqrt{2 \Omega^{2}+\omega_{x}^{2}+\omega_{y}^{2}-2 \epsilon \sqrt{\left(\omega_{x}^{2}-\Omega^{2}\right)\left(\omega_{y}^{2}-\Omega^{2}\right)}}}{2}=\frac{\omega_{+}-\epsilon \omega_{-}}{2},
$$

where $\epsilon$ is the sign parameter equal to 1 for slow rotations and equal to -1 for fast rotations. It follows from these definitions that for slow rotations $\Omega_{1}>\Omega_{2}$ and for fast rotations $\Omega_{2}>\Omega_{1}$. By inverting the formulas (24) we obtain the trap frequencies expressed in terms of $\Omega_{2}$ and $\Omega_{1}$

$$
\begin{aligned}
& \omega_{x}=\sqrt{\Omega_{1}^{2}+\Omega_{2}^{2}-\Omega^{2}+2 \sqrt{\left(\Omega_{1}^{2}-\Omega^{2}\right)\left(\Omega_{2}^{2}-\Omega^{2}\right)}}, \\
& \omega_{y}=\sqrt{\Omega_{1}^{2}+\Omega_{2}^{2}-\Omega^{2}-2 \sqrt{\left(\Omega_{1}^{2}-\Omega^{2}\right)\left(\Omega_{2}^{2}-\Omega^{2}\right)}} .
\end{aligned}
$$

We shall build the generating function according to the formula (21), choosing the ground state as the envelope function. Owing to our special choice of the rotation axis, the generating function in three dimensions is a product of the generating function in the $x$ and $y$ variables and the generating function for the one-dimensional oscillator in the $z$ variable

$$
\phi_{\boldsymbol{k}}(\boldsymbol{r}, t)=\phi_{k_{x} k_{y}}(x, y, t) \phi_{k_{z}}(z, t) .
$$

The last part has been already obtained in Ref. [10] and it has the form (we leave out normalization constants for they play no role in our considerations)

$$
\begin{aligned}
& \phi_{k_{z}}(z, t)=\exp \left[-\frac{\mathrm{i} \omega_{z} t}{2}-\mathrm{ie}^{-\mathrm{i} \omega_{z} t} \frac{\hbar k_{z}^{2}}{2 m \omega_{z}} \sin \left(\omega_{z} t\right)\right] \exp \left(-\frac{m \omega_{z}}{2 \hbar} z^{2}\right) \\
& \quad \times \exp \left(\mathrm{ie}^{-\mathrm{i} \omega_{z} t} k_{z} z\right) .
\end{aligned}
$$

The generating function in the $x$ and $y$ variables has the same general structure. It is also a product of the time-dependent phase factor, the time-independent Gaussian, and the phase factor that is linear in the coordinates

$$
\phi_{k_{x} k_{y}}(x, y, t)=\exp (\mathrm{i} \varphi(t)) \exp \left(-\frac{m}{2 \hbar} \boldsymbol{r}_{\perp} \cdot \hat{M} \cdot \boldsymbol{r}_{\perp}\right) \exp \left(\mathrm{i} \boldsymbol{a}(t) \cdot \boldsymbol{r}_{\perp}\right),
$$

where $\hat{M}$ is a $2 \times 2$ symmetric constant matrix, $\boldsymbol{a}(t)$ is a two-dimensional vector, $\varphi(t)$ is a phase, and $r_{\perp}$ is the projection of the vector $r$ into the $x y$ plane. Upon substituting this ansatz into the Schrödinger equation (20), one obtains a set of algebraic equations for the components of the matrix $\hat{M}$, a first-order linear differential equation for the vector $a(t)$ and an expression for the derivative of the phase $\varphi(t)$,

$$
\begin{aligned}
& \hat{M}^{2}+\mathrm{i}[\hat{\Omega}, \hat{M}]-\hat{V}=0, \\
& \frac{\mathrm{d} \boldsymbol{a}(t)}{\mathrm{d} t}=(-\mathrm{i} \hat{M}+\hat{\Omega}) \boldsymbol{a}(t), \\
& \frac{\mathrm{d} \varphi(t)}{\mathrm{d} t}=-\frac{\operatorname{Tr}\{\hat{M}\}}{2}-\hbar \frac{\boldsymbol{a}(t)^{2}}{2 m},
\end{aligned}
$$

where $\hat{\Omega}=\{\{0, \Omega\},\{-\Omega, 0\}\}$ is the matrix representing the rotation and $\hat{V}=$ $\left\{\left\{\omega_{x}^{2}, 0\right\},\left\{0, \omega_{y}^{2}\right\}\right\}$ is the matrix of the potential energy divided by $m$. The initial values are $a(0)=k_{\perp}, \varphi(0)=0$. Equations (29) may be successively solved starting 
with the equation for $\hat{M}$. The solution for the matrix $\hat{M}$ is

$$
\hat{M}=\left[\begin{array}{cc}
\Omega_{1}(1+\kappa) & \mathrm{i} \Omega \kappa \\
\mathrm{i} \Omega \kappa & \Omega_{1}(1-\kappa)
\end{array}\right],
$$

where

$$
\kappa=\epsilon \sqrt{\frac{\Omega_{2}^{2}-\Omega^{2}}{\Omega_{1}^{2}-\Omega^{2}}}=\epsilon \sqrt{\frac{2 \Omega^{2}-\omega_{x}^{2}-\omega_{y}^{2}+2 \epsilon \sqrt{\left(\omega_{x}^{2}-\Omega^{2}\right)\left(\omega_{y}^{2}-\Omega^{2}\right)}}{2 \Omega^{2}-\omega_{x}^{2}-\omega_{y}^{2}-2 \epsilon \sqrt{\left(\omega_{x}^{2}-\Omega^{2}\right)\left(\omega_{y}^{2}-\Omega^{2}\right)}}} .
$$

Note that since $\left(\Omega_{1}^{2}-\Omega^{2}\right)\left(\Omega_{2}^{2}-\Omega^{2}\right)=\left(\omega_{x}^{2}-\omega_{y}^{2}\right)^{2}, \kappa$ is always real. In addition, the values of $\kappa$ at the boundaries of the stability region $\omega_{y}$ and $\omega_{x}$ are equal to 1 and -1 , respectively and the derivative of $\kappa$ with respect to $\Omega$ in the stability regions is always positive

$$
\frac{d \kappa}{d \Omega}=\frac{2 \Omega \epsilon \kappa}{\sqrt{\left(\omega_{x}^{2}-\Omega^{2}\right)\left(\omega_{y}^{2}-\Omega^{2}\right)}}>0 .
$$

Typical behavior of $\kappa$ as a function of $\Omega$ is shown in Fig. 5. Thus, the absolute value of $\kappa$ inside the stability region is less than 1 . Therefore, the matrix $\hat{M}$ always has a

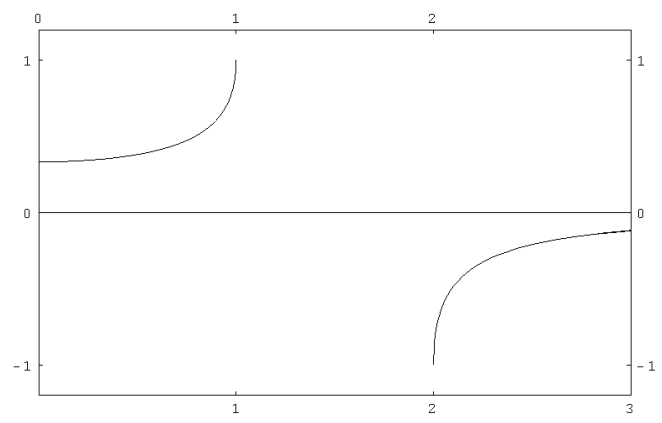

Fig. 5. The plot of $\kappa$ as a function of $\Omega$ for the values of the frequencies (in arbitrary units) $\omega_{y}=1$ and $\omega_{x}=2$.

positive definite real part and hence it defines a bounded Gaussian wave function. Once $\hat{M}$ has been determined, the remaining two equations may be solved. We shall write down the solutions with the physical constants $\hbar$ and $m$ inserted

$$
\begin{aligned}
& \boldsymbol{a}(t)=\exp [(-\mathrm{i} \hat{M}+\hat{\Omega}) t] \cdot \boldsymbol{k}_{\perp}=\mathrm{e}^{-\mathrm{i} \Omega_{1} t}\left[\cos \left(\Omega_{2} t\right)-\mathrm{i}(\boldsymbol{\sigma} \cdot \boldsymbol{b}) \sin \left(\Omega_{2} t\right)\right] \cdot \boldsymbol{k}_{\perp}, \\
& \varphi(t)=\frac{\hbar}{2 m} \boldsymbol{k}_{\perp} \cdot\left[f(t)+g(t) \sigma_{x}+h(t) \sigma_{z}\right] \cdot \boldsymbol{k}_{\perp}-\Omega_{1} t
\end{aligned}
$$

where $\sigma_{x}, \sigma_{y}$, and $\sigma_{z}$ are the standard Pauli matrices (introduced here only for convenience; there is no spin in our problem), $\boldsymbol{b}$ is the following complex unit vector 


$$
b=\frac{1}{\Omega_{2}}\left\{\mathrm{i} \kappa \Omega,-\Omega, \kappa \Omega_{1}\right\},
$$

and $f, g$, and $h$ are three complex functions of time

$$
\begin{aligned}
& f(t)=-\frac{\mathrm{i}\left(\Omega_{1}^{2}-\Omega^{2}\right)}{2 \Omega_{1}\left(\Omega_{1}^{2}-\Omega_{2}^{2}\right)}+\mathrm{ie}^{-2 \mathrm{i} \Omega_{1} t} \\
& \times \frac{\Omega_{1}\left(\Omega_{2}^{2}-\Omega^{2}\right)\left[\Omega_{1} \cos \left(2 \Omega_{2} t\right)+\mathrm{i} \Omega_{2} \sin \left(2 \Omega_{2} t\right)\right]+\Omega^{2}\left(\Omega_{1}^{2}-\Omega_{2}^{2}\right)}{2 \Omega_{1} \Omega_{2}^{2}\left(\Omega_{1}^{2}-\Omega_{2}^{2}\right)}, \\
& g(t)=\kappa \mathrm{e}^{-2 \mathrm{i} \Omega_{1} t} \frac{\Omega \sin ^{2}\left(\Omega_{2} t\right)}{\Omega_{2}^{2}}, \\
& h(t)=\frac{\mathrm{i} \kappa\left(\Omega_{1}^{2}-\Omega^{2}\right)}{2 \Omega_{1}\left(\Omega_{1}^{2}-\Omega_{2}^{2}\right)}-\mathrm{i} \kappa \mathrm{e}^{-2 \mathrm{i} \Omega_{1} t} \\
& \times \frac{\Omega_{1}\left[\Omega_{1}\left(\Omega_{2}^{2}-\Omega^{2}\right) \cos \left(2 \Omega_{2} t\right)+\mathrm{i} \Omega_{2}\left(\Omega_{1}^{2}-\Omega^{2}\right) \sin \left(2 \Omega_{2} t\right)\right]+\Omega^{2}\left(\Omega_{1}^{2}-\Omega_{2}^{2}\right)}{2 \Omega_{1} \Omega_{2}^{2}\left(\Omega_{1}^{2}-\Omega_{2}^{2}\right)} .
\end{aligned}
$$

We illustrate our results with two simple configurations of vortex lines. The first example involves two parallel vortex lines with the same circulation. These vortex lines remain straight and parallel all the time (cf. Fig. 6). In the second example, two vortex lines have opposite circulation. In this case the time evolution is completely different; both vortex lines become curved and move around in a complicated fashion (cf. Fig. 7).

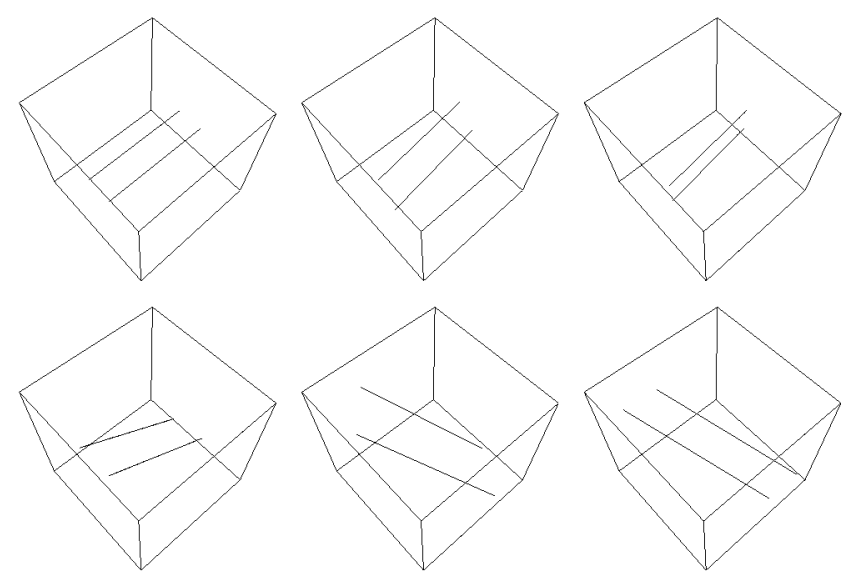

Fig. 6. Two vortex lines with the same circulation in a rotating trap. 


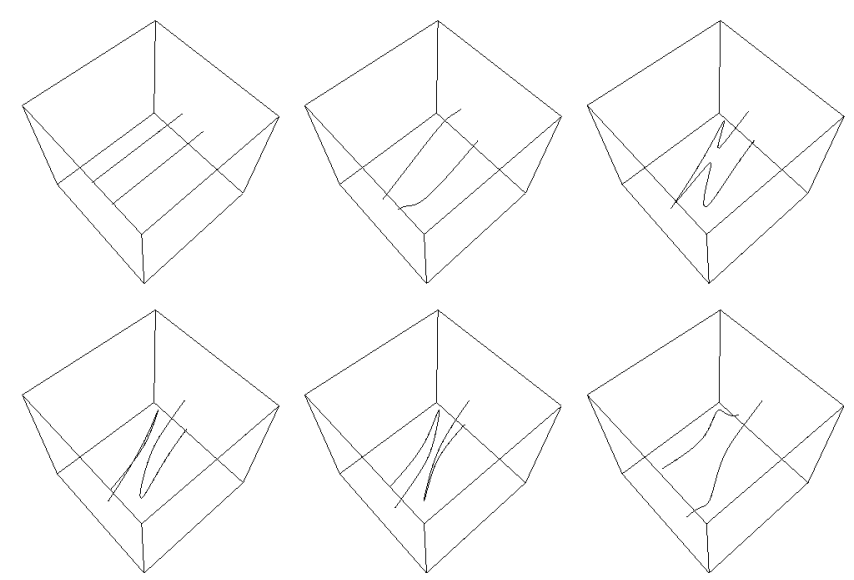

Fig. 7. Two vortex lines with opposite circulation in a rotating trap.

\section{Conclusions}

The main result of this paper is the extension of our method of generating functions to the case of a rotating harmonic trap. Such traps are now often used in the experiments on the Bose-Einstein condensates. We have derived a complete expression for the generating function in the case when the rotation axis coincides with one of the principal axes of the trap. In order to show its practical value, we have used this expression in a very simple case to find the motion of two vortex lines. Our generating function may be used to study systematically the motion of more complicated vortex structures. The serious limitation of this method in applications to realistic Bose-Einstein condensates is our use of the linear approximation to the wave equation. However, as it was argued in Ref. [11], this approximation might be adequate to describe the motion of vortices.

\section{References}

[1] O. Madelung, Z. Phys. 40, 342 (1926).

[2] Aristotle, De Caelo (On the Heavens), Book II, Ch. XIII.

[3] R. Descartes, Principia Philosophiae, Amsterdam 1644.

[4] W. Thomson (Lord Kelvin), Philos. Mag., 34, 15 (1867).

[5] G. Helmholtz, Crelles J., 55, 25 (1858) (English translation P.G. Tait, Philos. Mag., 33, 485 (1867).

[6] P.G. Saffman, Vortex Dynamics, Cambridge University, Cambridge 1992.

[7] J.F. Nye, M.V. Berry, Proc. R. Soc. Lond. A 336, 165 (1974).

[8] M.V. Berry, in: Les Houches Lecture Series, Session 35, Eds. R. Balian, M. Kléman, J.-P. Poirier, North-Holland, Amsterdam 1981, p. 453.

[9] M.V. Berry, M.R. Dennis, Proc. R. Soc. Lond. A 457, 2251 (2001). 
[10] I. Białynicki-Birula, Z. Białynicka-Birula, C. Śliwa, Phys. Rev. A 61, 032110 (2000).

[11] I. Białynicki-Birula, Z. Białynicka-Birula, Phys. Rev. A, in print.

[12] C. Śliwa, I. Białynicki-Birula, Z. Białynicka-Birula, T. Młoduchowski, in: Proc. IUTAM Symp., in print.

[13] I. Białynicki-Birula, Z. Białynicka-Birula, Phys. Rev. Lett. 78, 2539 (1997).

[14] I. Białynicki-Birula, J.H. Eberly, M. Kalinski, Phys. Rev. Lett. 73, 1777 (1994). 